\title{
Estudo da influência dos padrões de emissão das espécies do plasma de carbonitretação nas propriedades superficiais de TiCN
}

\author{
Influence of the emission patterns of \\ species in a carbonitriding plasma \\ on the surface properties of TiCN
}

\author{
Antonio Nunes Filho ${ }^{1}$, Danilo Cavalcanti Braz ${ }^{1}$, \\ Ruth Hinrichs ${ }^{2}$,Marcos A. Z. Vasconcellos ${ }^{2}$, \\ Ricardo C. S. Rocha ${ }^{1}$, Clodomiro Alves Jr. ${ }^{1}$
}

\footnotetext{
${ }^{1}$ Universidade Federal do Rio Grande do Norte - UFRN, Salgado Filho, CEP: 59072-970, Natal, Rio Grande do Norte, RN.

e-mail: antonio.jr.materiais@gmail.com, cavalte@yahoo.com.br, ricardocesarsr@gmail.com.br

${ }^{2}$ Universidade Federal do Rio Grande do Sul - UFRGS, CEP: 91501-970, Porto Alegre, RS.

e-mail: ruth.hinrichs@ufrgs.br,marcos@if.ufgs.br
}

\section{RESUMO}

As intensidades de emissão das espécies $\mathrm{N}_{2}^{+}, \mathrm{H}, \mathrm{C}, \mathrm{NH}, \mathrm{N}_{2}, \mathrm{Ar}$ e $\mathrm{CN}$ do plasma de carbonitretação (usando os gases $\mathrm{Ar}+\mathrm{N}_{2}+\mathrm{CH}_{4}$ ) foram medidas por espectroscopia de emissão óptica (OES) em pressão e temperatura constantes $\left(2 \mathrm{mbar}\right.$ e $500{ }^{\circ} \mathrm{C}$ ) variando o fluxo de $\mathrm{CH}_{4}$. Em seguida, amostras de Ti comercialmente puro (Ti $\mathrm{CP})$ foram carbonitretadas em plasma nas mesmas condições do estudo por OES. O objetivo desse trabalho é avaliar a influência das espécies presentes no plasma, detectadas por seus padrões de emissão, na composição de fases superficiais, nas características topográficas e no perfil de microdureza do revestimento de TiCN. Foi observado que o aumento da concentração de $\mathrm{CH}_{4}$ no plasma gerou as espécies $\mathrm{H}$ (dissociado do $\mathrm{CH}_{4}$ ) $\mathrm{N}_{2}{ }^{+}, \mathrm{C}$, NH e CN. A evolução contínua de $\mathrm{CN}$ e uma queda progressiva na espécie $\mathrm{N}_{2}{ }^{+}$influenciaram a composição da superfície e as propriedades aqui avaliadas. A análise de difração de raios $\mathrm{X}$ em ângulo rasante revelou a presença de nitreto e carbonitreto de titânio na superfície com composição química correlacionada com as alterações das intensidades de emissão das espécies $\mathrm{CN} \mathrm{e} \mathrm{N}_{2}^{+}$. As topografias das amostras, avaliadas por microscopia de força atômica, apresentaram aumento nos parâmetros de rugosidade comparados com a amostra padrão. A rugosidade $\mathrm{Ra}$ aumentou com o aumento da intensidade de emissão óptica de $\mathrm{CN}$ e redução de $\mathrm{N}_{2}{ }^{+}$. Com auxílio da espectroscopia micro-Raman foi encontrado carbono não reagido com o titânio na forma de um filme que se desenvolveu com o aumento da intensidade da linha de emissão do CN. Este filme pode, hipoteticamente, ter sido o responsável pela queda progressiva da dureza superficial do revestimento.

Palavras-chave: titânio, carbonitretação a plasma, OES, GIXRD, espectroscopia Raman, microdureza.

\section{ABSTRACT}

The optical emission intensities of the species $\mathrm{N}_{2}{ }^{+}, \mathrm{H}, \mathrm{C}, \mathrm{NH}, \mathrm{N}_{2}, \mathrm{Ar}$ e $\mathrm{CN}$ in a carbonitriding plasma (using $\mathrm{Ar}+\mathrm{N}_{2}+\mathrm{CH}_{4}$ gases) were studied with optical emission spectroscopy in an atmosphere of constant pressure and temperature, changing the $\mathrm{CH}_{4}$ flow. Afterwards a commercially pure Ti was exposed to a carbonitriding plasma in similar conditions. The objective of this work is to evaluate the influence of the gaseous species on the superficial properties (chemical composition, topography, and microhardness profile) of the TiCN layer. When the $\mathrm{CH}_{4}$ flow was increased, the species $\mathrm{H}, \mathrm{N}_{2}{ }^{+}, \mathrm{C}, \mathrm{NH}$, and $\mathrm{CN}$ were observed. The continuous increase of $\mathrm{CN}$ and a progressive reduction of $\mathrm{N}_{2}{ }^{+}$were detected. Grazing incidence X-ray diffraction revealed 
titanium nitride and carbonitride with variable chemical compositions, related to the changes observed in the emission patterns of the species $\mathrm{CN}$ and $\mathrm{N}_{2}{ }^{+}$. The topography, evaluated by atomic force microscopy, showed that the roughness increased when $\mathrm{CN}$ increased and $\mathrm{N}_{2}{ }^{+}$decreased. Micro-Raman spectroscopy showed unreacted carbon on the surface that developed with de increase of the $\mathrm{CN}$ emission. This carbon film may be responsible for the progressive decrease of surface hardness.

Keywords: titanium, plasma carbonitriding, OES, GIXRD, Raman spectroscopy, microhardness.

\section{INTRODUÇÃO}

O desempenho de ferramentas de corte e utensílios de aplicação biomédica que atuam em ambientes agressivos é influenciado pelas propriedades físico-químicas superficiais. Devido a isso, revestimentos a base de TiN, TiC e TiCN são muito utilizados nesses ambientes corrosivos e com carregamento mecânico devido as suas propriedades, tais como: alta dureza, baixo coeficiente de atrito, boa resistência à corrosão e biocompatibilidade [1-3].

$\mathrm{O} \mathrm{TiC}_{\mathrm{x}} \mathrm{N}_{1-\mathrm{x}}$ (onde $0<\mathrm{x}<1$ ) é descrito na literatura como uma solução sólida de TiN + TiC. Portanto, este filme incorpora as características das duas fases presentes $[4,5]$, conforme a relação $\mathrm{C} / \mathrm{N}$ que elas apresentam. A versatilidade do sistema deve-se ao fato de oTiC e o TiN serem isoestruturais e completamente miscíveis. Os átomos de $\mathrm{C}$ e $\mathrm{N}$ competem pelos espaços intersticiais da rede do titânio. Em geral o TiN gera boa tenacidade e adesão, o que compensa a fragilidade do TiC, porém este último contribui com maior dure$\mathrm{za}$, resistência ao desgaste e menor coeficiente de atrito [ $[\underline{6}, \underline{7}]$.

As condições dos processos a plasma têm influencia nas características dos revestimentos. O entendimento e a otimização dos parâmetros requerem informações dos mecanismos de formação da camada, os quais são influenciados pelas características do plasma. Átomos e moléculas excitadas tem relevância no crescimento das camadas devido sua alta atividade química [ㅇ-11]. Portanto é importante avaliar o processo sem perturbá-lo, observando variações dos parâmetros durante a formação da camada ou alcançar um regime estacionário que garanta reprodutibilidade máxima do processo de fabricação numa escala industrial [12].

Para este fim o diagnóstico do plasma por espectroscopia de emissão óptica (OES) é vantajoso pela sua simplicidade. As linhas atômicas e moleculares de várias espécies podem ser detectadas e estudadas, relacionando suas intensidades de emissão com as propriedades encontradas na camada nitretada. Essa técnica possibilita obter informações sobre os padrões de fragmentação de gases [11, 13].

Este trabalho traz um estudo dos padrões de emissão das espécies $\mathrm{N}_{2}, \mathrm{NH}, \mathrm{Ar}, \mathrm{N}_{2}^{+}, \mathrm{H}, \mathrm{C}$ e $\mathrm{CN}$ do plasma de carbonitretação, usando atmosfera de $\mathrm{Ar}+\mathrm{N}_{2}+\mathrm{CH}_{4}$ em pressão e temperatura constantes (2mbar e $500{ }^{\circ} \mathrm{C}$ ), variando as concentrações dos gases reagentes. $\mathrm{O}$ tratamento a plasma nessas diversas atmosferas permitiu o revestimento de vários substratos com filmes de TiCN com diferentes razões $\mathrm{C} / \mathrm{N}$. Os padrões de emissão foram estudados e relacionados com as propriedades superficiais do TiCN. As amostras foram caracterizadas por difração de raios X em ângulo rasante (GIXRD), espectroscopia micro-Raman, microscopia de força atômica (AFM) e microdureza.

\section{MATERIAIS E MÉTODOS}

O plasma estudado teve seu fluxo de $\mathrm{CH}_{4}$ variado de $0-4 \mathrm{sccm}$, enquanto foram mantidos constantes os fluxos de $\mathrm{Ar}$ e $\mathrm{N}_{2}$ (1 e $3 \mathrm{sccm}$ respectivamente), com o objetivo de obter uma correlação entre as linhas de emissão e as propriedades superficiais das amostras carbonitretadas. As linhas de emissão foram monitoradas nas condições semelhantes às que foram usadas para realizar o tratamento termoquímico (tabela 1). O diagnóstico de plasma foi efetuado usando um espectrômetro de emissão óptica (OES ActonSpectrapro 2500i) com comprimento focal de $500 \mathrm{~mm}$. As linhas de emissão foram adquiridas numa faixa de $300-800 \mathrm{~nm}$.

Tabela 1: Parâmetros de processos para fabricação de TiCN em temperatura $\left(500{ }^{\circ} \mathrm{C}\right)$ e pressão constante $(2,0$ mbar $)$.

Fluxo de gás (sccm)

\begin{tabular}{c|c|c|c|c|c}
\hline AMOSTRA & $\mathbf{N}_{\mathbf{2}}$ & $\mathbf{A r}$ & $\mathbf{C H}_{\mathbf{4}}$ & $\begin{array}{c}\text { TENSÃO } \\
(\mathbf{V})\end{array}$ & $\begin{array}{c}\text { CORRENTE } \\
(\mathbf{A})\end{array}$ \\
\hline TiCNI & 3 & 1 & 1 & 777 & 0,09 \\
\hline TiCNII & 3 & 1 & 2 & 898 & 0,09 \\
\hline TiCNIII & 3 & 1 & 3 & 1060 & 0,09 \\
\hline
\end{tabular}


Discos de Ti cp com $15 \mathrm{~mm}$ de diâmetro e $2 \mathrm{~mm}$ de espessura foram lixados e polidos em solução de peróxido de hidrogênio $\left(\mathrm{H}_{2} \mathrm{O}_{2}\right)$ e sílica coloidal a $40 \%$, até atingir uma rugosidade $\mathrm{Ra} \sim 0.51 \mathrm{~nm}$, e sucessivamente limpas com detergente enzimático (RIOZIME® IV-E NEUTRO gold, fabricado pela RIOQUÍMICA), acetona e água destilada, em banho de ultrassom durante $10 \mathrm{~min}$. Para os tratamentos termoquímicos de carbonitretação a plasma, foi utilizado um reator de aço inox de geometria cilíndrica com $300 \mathrm{~mm}$ de diâmetro e $300 \mathrm{~mm}$ de altura, fechado por dois flanges e vedado com gaxetas de viton com perfil em "L". O flange da extremidade inferior dispõe de conexões de gases, sensor de pressão, saída para sistema de vácuo e cátodo (haste cilíndrica em aço inoxidável com $15 \mathrm{~mm}$ de diâmetro e $150 \mathrm{~mm}$ de comprimento). Preso ao cátodo está o porta-amostras. Um termopar de cromel-alumel foi inserido no interior do cátodo até o porta-amostras para medição da temperatura de referência do processo. Na parte lateral do reator existe uma janela de quartzo que permite visualizar o plasma. Para a alimentação dos gases foram utilizados fluxômetros digitais MKS (modelo 247). O plasma é sustentado por uma fonte de tensão contínua ajustável (0-1200 V). A figura 1 ilustra o set-up experimental e o esquema de posicionamento da fibra óptica durante o diagnóstico de plasma por OES. Os parâmetros do processo usado para a fabricação das amostras de TiCN e a nomenclatura das mesmas estão na tabela 1 .

As linhas de emissão das espécies observadas neste estudo estão listadas na tabela 2, de acordo com o National Institute of Standards and Technology (NIST) [14] para as espécies atômicas e em acordo com PEARSE e GAYDON [15] para as espécies moleculares. $\mathrm{CH}, \mathrm{CN}, \mathrm{C}, \mathrm{H}, \mathrm{N}_{2}^{+}$e $\mathrm{N}_{2}$ têm um importante papel na carbonitretação a plasma [16], porém eventualmente algumas moléculas orgânicas, tipo $\mathrm{C}_{\mathrm{x}} \mathrm{H}_{\mathrm{y}}(\mathrm{x}>1, \mathrm{y}>1)$ podem ter estado presentes e não terem sido identificadas. As linhas de emissão mais intensas desses compostos orgânicos estão na faixa de UV, não alcançada pela faixa espectral do equipamento []]. Neste trabalho foram consideradas as emissões de $\mathrm{H} \alpha, \mathrm{CN}, \mathrm{C}, \mathrm{NH}, \mathrm{Ar}, \mathrm{N}_{2}^{+}$e $\mathrm{N}_{2}$.

Tabela 2: Linhas de emissão espectral e as bandas de transição das espécies selecionadas.

\begin{tabular}{c|c|c}
\hline ESPÉCIE & TRANSIÇÃO & $\begin{array}{c}\text { COMPRIMENTO } \\
\text { DE ONDA (nm) }\end{array}$ \\
\hline $\mathrm{H} \alpha$ & Séries de Balmer $3 \mathrm{~d}-2 \mathrm{p}$ & 656,3 \\
\hline $\mathrm{Ar}$ & $3 \mathrm{~s}^{2} 3 \mathrm{p}^{5}\left({ }^{2} \mathrm{P}^{\circ}{ }_{1 / 2}\right) 4 \mathrm{p}-3 \mathrm{~s}^{2} 3 \mathrm{p}^{5}\left({ }^{2} \mathrm{P}^{\circ}{ }_{1 / 2}\right) 4 \mathrm{~s}$ & 750,3 \\
\hline $\mathrm{N}_{2}^{+}$ & $\mathrm{B}^{2} \Sigma_{\mathrm{u}}^{+}-\mathrm{X}^{2} \Sigma_{\mathrm{g}}{ }^{+}(0-0)$ & 391,4 \\
\hline $\mathrm{CN}$ & $\mathrm{B}^{2} \Sigma-\mathrm{X}^{2} \Sigma(0-0)$ & 388,4 \\
\hline $\mathrm{C}$ & $2 \mathrm{~s}^{2} 2 \mathrm{p} 4 \mathrm{p}^{1} \mathrm{D} 2-2 \mathrm{~s}^{1} 2 \mathrm{p} 3 \mathrm{~s}^{1} \mathrm{P}^{\mathrm{o}} 1$ & 505,2 \\
\hline $\mathrm{NH}$ & $\mathrm{A}^{3} \Pi-\mathrm{X}^{3} \Sigma(0-0)$ & 336 \\
\hline $\mathrm{N}_{2}$ & $\mathrm{C}^{3} \Pi_{\mathrm{u}}-\mathrm{B}^{3} \Pi_{\mathrm{g}}(0-1)$ & 357,7 \\
\hline
\end{tabular}
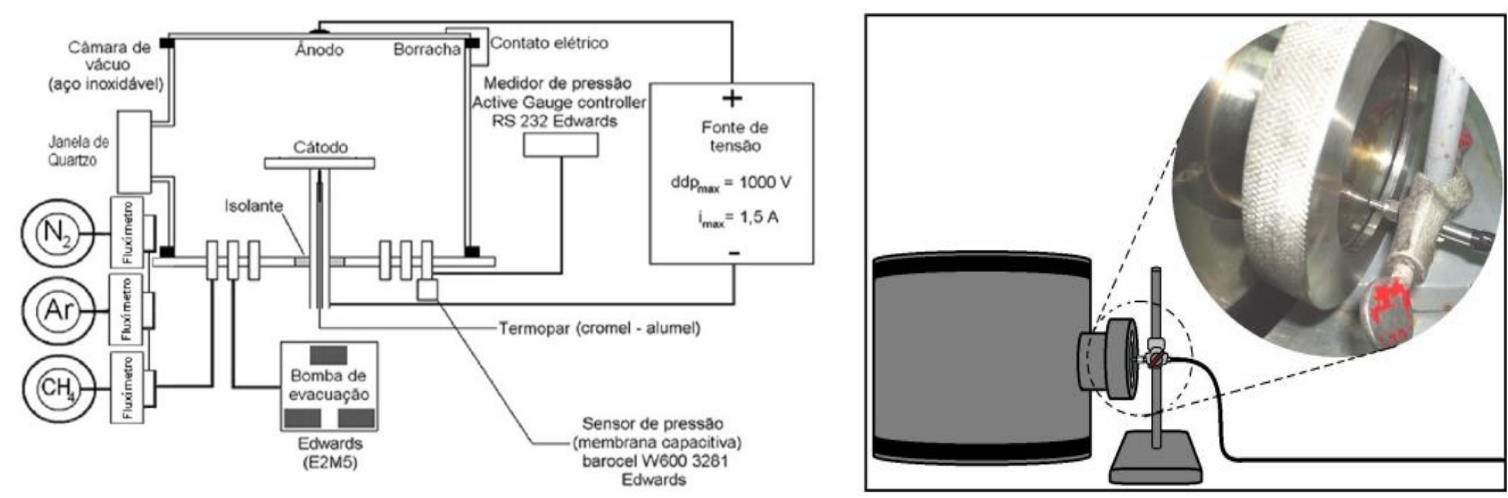

(a)(b) 
Figura 1: (a) Esquema do reator de plasma usado na produção de TiCN e (b) posicionamento da fibra óptica na janela de quartzo para análise do plasma por OES .

As superfícies foram caracterizadas por GIXRD com incidência de $0.5^{\circ}$, espectroscopia micro-Raman,

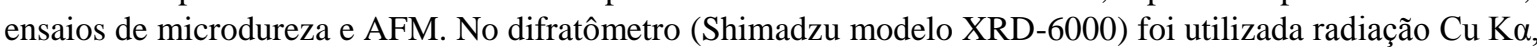
gerada por um tubo operado com $40 \mathrm{kV}$ e $30 \mathrm{~mA}$, numa varredura em $2 \theta$ de $33.00-45.00^{\circ}$. Os padrões de difração foram comparados com as fichas padrão PDF-ICDD (Powder diffraction file of the International Center for Diffraction Data). A espectroscopia micro-Raman foi realizada usando um laser de He-Ne (comprimento de onda de $632.8 \mathrm{~nm}$ ), com feixe de $\sim 3 \mu \mathrm{m}$ de diâmetro, apontado para o centro da amostra. $\mathrm{O}$ tempo de aquisição de dados foi de $100 \mathrm{~s}$. A potência do laser foi diminuída para evitar modificações na superfície. Os ensaios de microdureza foram realizados utilizando o Nanoindenter ${ }^{\circledR X P}$ da MTS Instruments. Para medida de microdureza foi utilizado o método de Oliver e Pharr e o programa Test Works 4 da MTS Systems Corporation. Nove endentações com espaçamento de $100 \mu \mathrm{m}$ entre elas, dispostas numa matriz 3x3 (figura 2) foram feitas com um penetrador Berkovich, com cargas de 0,2 $\mathrm{mN}$ a $400 \mathrm{mN}$, utilizando 12 ciclos de carga-descarga, obtendo um gráfico da carga aplicada em função da profundidade com as suas respectivas barras de erros. A topografia e a rugosidade foram analisadas por um microscópio de força atômica (AFM Shimadzu, SPM 9600), equipado com um scanner de variação máxima de $55 \mu \mathrm{m}$ nas direções x e y e $13 \mu \mathrm{m}$ na direção z. Os perfis de rugosidade avaliados foram à rugosidade média (Ra), altura máxima média do perfil (Rz), altura máxima do perfil do pico (Rp) e a altura máxima do perfil do vale (Rv). As imagens foram obtidas em um tamanho de $5 \times 5 \mu \mathrm{m}$ com uma resolução de 256x256 pontos.

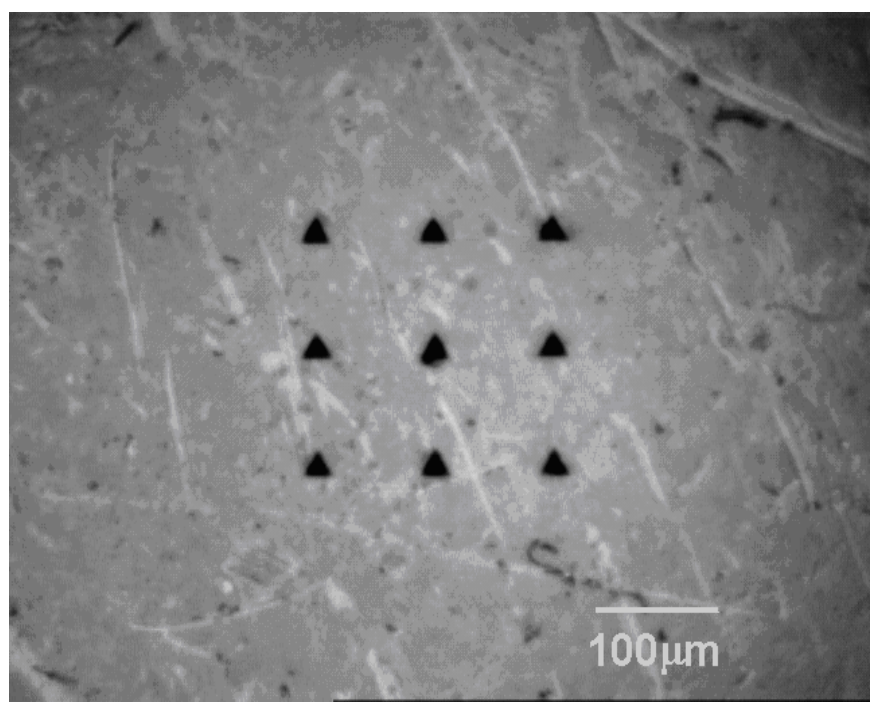

Figura 2: Micrografia de MEV da disposição das impressões do ensaio de microdureza organizados como uma matriz $3 \times 3$.

\section{RESULTADOS}

As intensidades relativas das linhas de emissão, normalizadas pela intensidade da linha de Ar, estão mostradas na figura 3 para as misturas de $1 \mathrm{sccm}$ de $\mathrm{Ar}, 3 \mathrm{sccm}$ de $\mathrm{N}_{2} \mathrm{com} \mathrm{0-4} \mathrm{sccm} \mathrm{de} \mathrm{CH}_{4}$. Como a pressão foi mantida constante, as variações das intensidades relativas podem ser associadas às concentrações das espécies presentes. Em $0 \mathrm{sccm}$ de $\mathrm{CH}_{4}$, os sinais de $\mathrm{CN}, \mathrm{C}, \mathrm{H} \alpha$ e $\mathrm{NH}$ são baixos, porém perceptíveis, mesmo sem $\mathrm{CH}_{4}$ no reator de plasma, devido ao gás atmosférico residual ou vapores de óleo provenientes da bomba de vácuo. Ao adicionar metano $(1 \mathrm{sccm})$, essas espécies passam a emitir em intensidades maiores. A dissociação contínua das moléculas de $\mathrm{N}_{2}$ e de $\mathrm{CH}_{4}$ formam produtos como $\mathrm{N}, \mathrm{H}$ e $\mathrm{C}$ atômicos e combinações moleculares dos mesmos. A queda de emissão do $\mathrm{N}_{2}$ e $\mathrm{N}_{2}{ }^{+}$em $2 \mathrm{sccm}$ de acréscimo de metano é refletida no aumento da produção de $\mathrm{C}$ e consequentemente nas demais espécies que são derivadas da dissociação do $\mathrm{CH}_{4}$. Já foi observado em estudos anteriores que a presença do nitrogênio aumenta a capacidade de dissociação do $\mathrm{CH}_{4}$ no plasma, a produção de $\mathrm{CN}$ e a intensidade de emissão de radicais contendo C [17]. O decréscimo de $\mathrm{N}_{2}{ }^{+}$é justificado pelo fato do mesmo estar reduzindo para $\mathrm{N}_{2}$ e decompondo-se para a formação de $\mathrm{CN}$ e $\mathrm{NH}$.

Além do mais, a proporção do gás $\mathrm{N}_{2}$ está diminuindo à medida que o fluxo de $\mathrm{CH}_{4}$ aumenta. $\mathrm{O} \mathrm{C}$ apresenta oscilação na sua intensidade semelhante ao $\mathrm{NH}$ e $\mathrm{N}_{2}$, o que pode ser devido à influência do nitro- 
gênio na dissociação do $\mathrm{CH}_{4}$ que reflete na produção de NH. LEGRAND et al. [18] estudaram um modelo de dissociação de $\mathrm{CH}_{4}$ em plasma de $\mathrm{CH}_{4}+\mathrm{N}_{2}$ no regime de pós-descarga e citaram algumas reações possíveis, são elas:

$$
\begin{aligned}
& \mathrm{CH}_{4}+e \rightarrow \mathrm{C}+4 \mathrm{H}+e \\
& \mathrm{CH}_{4}+\mathrm{N}_{2}{ }^{*} \rightarrow \mathrm{CH}_{3}+\mathrm{H}+\mathrm{N}_{2} \\
& \mathrm{CN}+\mathrm{N} \rightarrow \mathrm{C}+\mathrm{N}_{2} \\
& \mathrm{CH}+\mathrm{N} \rightarrow \mathrm{CN}+\mathrm{H} \\
& \mathrm{CH}_{3}+\mathrm{N} \rightarrow \mathrm{CH}_{2}+\mathrm{NH}
\end{aligned}
$$

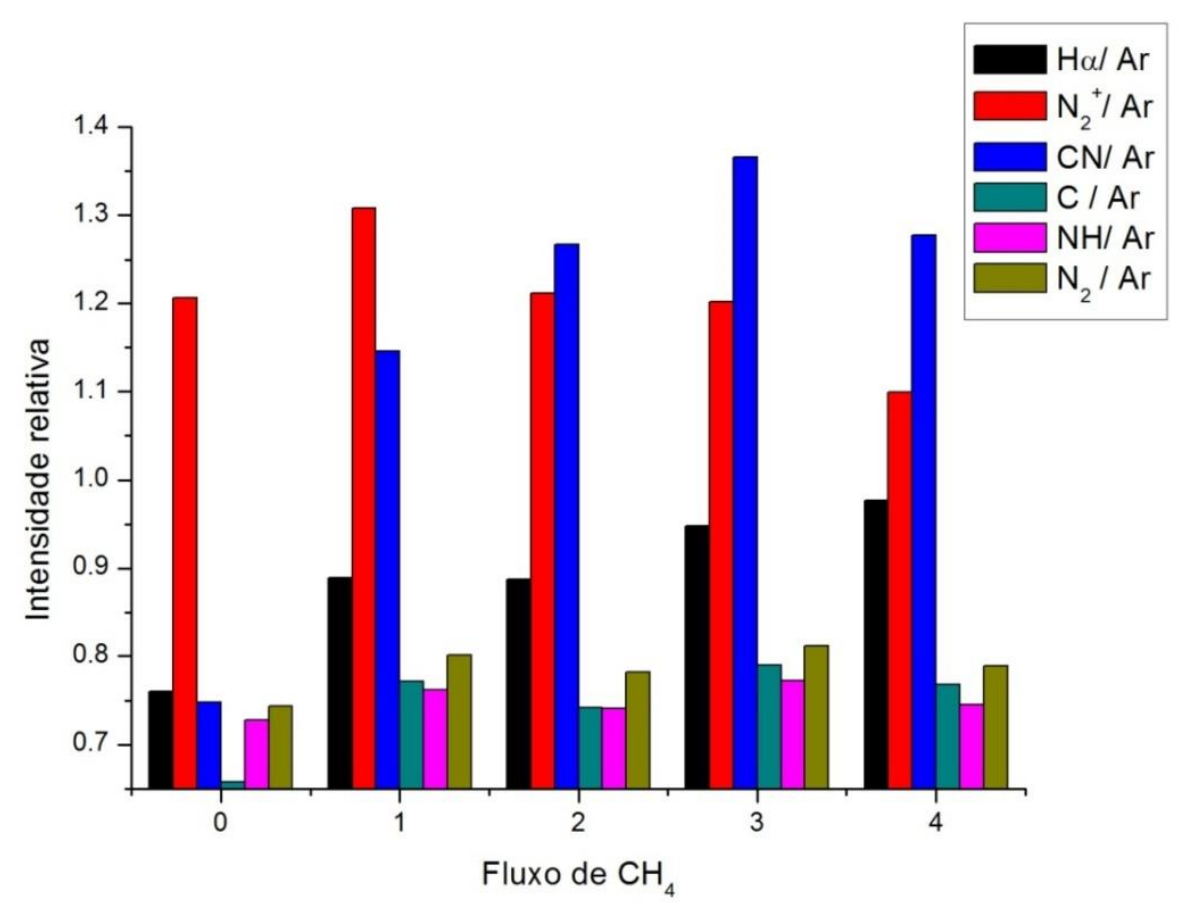

Figura 3: Intensidades de emissão das espécies (relativas ao sinal de $\mathrm{Ar}$ ), em função da variação do fluxo de $\mathrm{CH}_{4}$.

A figura 4a exibe a análise de difração de raios X das amostras de TiCN I-III em comparação com uma amostra de Ti cp padrão (polido). Os picos do $\alpha$-Ti estão deslocados nas amostras de TiCN devido à presença de tensões residuais gerados pela presença de $\mathrm{C}$ e $\mathrm{N}$ nos interstícios [19].

Devido ao gradiente de composição com a profundidade, ocorre o alargamento dos picos, principalmente, dos nitretos e carbonitretos. Fazendo a decomposição do pico na faixa de $41^{\circ}$ a $44^{\circ}$ usando funções gaussianas (figura $4 \mathrm{~b}$ ), pode-se observar que a fase TiN está mais presente na condição em que $\mathrm{N}_{2}{ }^{+}$possui maior intensidade, ou seja, quando o fluxo de $\mathrm{CH}_{4}$ é de apenas $1 \mathrm{sccm}$. Por outro lado, a presença de $\mathrm{CN}$ promove a formação de TiCN.

Os picos que são referentes ao TiN e TiCN estão alargados nos planos (111) e (200). Esse alargamento aumenta com a queda da emissão de $\mathrm{N}_{2}{ }^{+} \mathrm{e}$ a evolução do CN. Este fenômeno foi atribuídoà tensão não homogênea, variações na composição e nas dimensões do cristalito [19]. A inserção de átomos na célula unitária gera uma distribuição não uniforme de tensões causando o alargamento de pico [20]. Os alargamentos desses picos podem estar relacionados à formação de fases não estequiométricas por causa da larga faixa de solubilidade do titânio com C e N intersticiais, tendo em vista que as fases TiN e TiC podem se formar em qualquer proporção na rede do Ti por serem fases [19]. O processo de carbonitretação a plasma empregado neste trabalho gerou uma camada de $\mathrm{TiCN}$ de composição química variável, devido à variação da concentração de $\mathrm{CH}_{4}$ no reator de plasma. A presença dos picos de $\alpha$-Ti (fase hexagonal) e as fases TiN e TiCN (que- 
são cúbicas) evidencia uma superfície composta de uma matriz de $\alpha$-Ti com precipitados de TiN e TiCN.

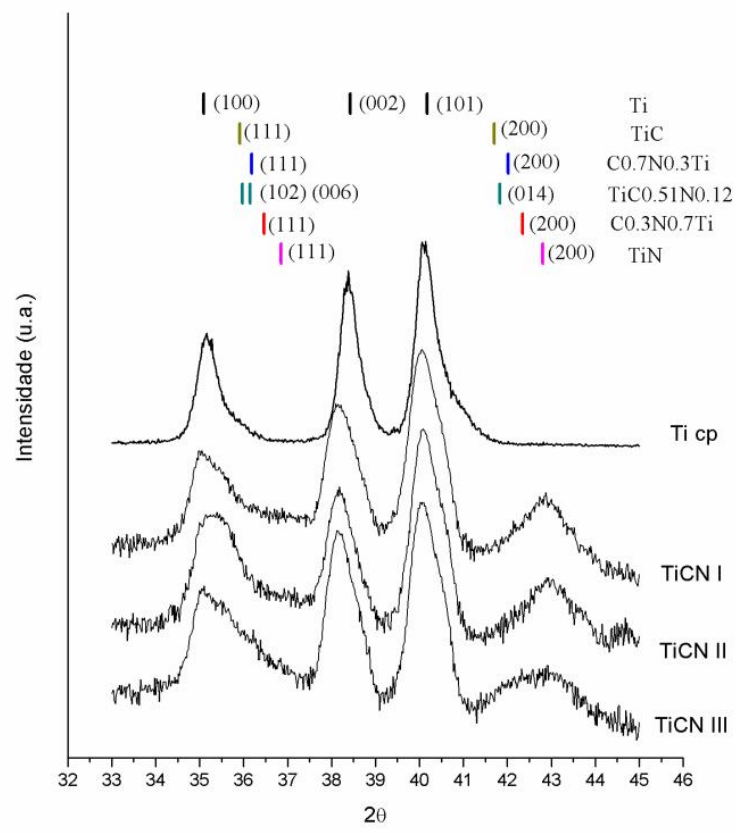

(a)

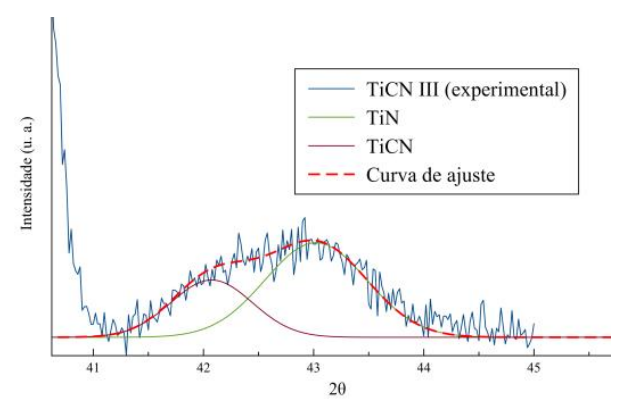

(b)

Figura 4: (a) Comparação dos padrões de difração de raios-X em ângulo rasante de $0,5^{\circ}$ das amostras de Ti cp e TiCN; (b) curvas de ajuste para a separação dos picos de TiN e TiCN.

Com objetivo de estudar a estrutura do carbono nas amostras de TiCN utilizou-se a espectroscopia micro-Raman. Foram adquiridos espectros na faixa de $900-1900 \mathrm{~cm}^{-1}$ onde estruturas compostas por carbono e grafite apresentam picos característicos. Esses picos são associados à banda D e a banda G [21]. PIMENTA et al. [22] observaram a posição desses picos na região $1350 \mathrm{~cm}^{-1}$ e $1582 \mathrm{~cm}^{-1}$, respectivamente, e informaram que tais espectros são referentes a carbono amorfo e grafite. O pico em $1582 \mathrm{~cm}^{-1}$ (banda G) pode ser associado aos modos de vibração do grafite cristalino, enquanto que o pico em $1350 \mathrm{~cm}^{-1}$ (banda D, desordem) é atribuído aos defeitos dos contornos dos cristais de grafite devido a seu tamanho finito. Este pico se torna mais intenso quanto menor forem os grãos do grafite, ou quanto mais desordenado for o cristalito. Observou-se a presença de carbono depositado na superfície das amostras de TiCN, não reagido com o Ti [23].

O estudo dos espectros Raman foi auxiliado pelo software Origin 8.0. Utilizaram-se 4 curvas (tipo Lorentzianas) para ajustá-los, de acordo com ZAIDA [24] (figura 5). Nessas curvas estão identificadas as bandas D e G (linhas cheias) que será o objeto de observação deste trabalho. As outras duas curvas (linhas pontilhadas) representam ligações tipo $\mathrm{sp}^{2}$ ou mistura entre $\mathrm{sp}^{2}$ e $\mathrm{sp}^{3}$ [24]. A tabela 3 exibe os dados da localização das bandas D e G (deslocamento Raman em $\left.\mathrm{cm}^{-1}\right)$, largura à meia altura $\left(\mathrm{FWHM}_{\mathrm{D}} \mathrm{e} \mathrm{FWHM}_{\mathrm{G}}\right)$, a áreas $\left(I_{D}\right.$ e $\left.I_{G}\right)$ e a relação $I_{D} / I_{G}$ referentes a esses ajustes.

Tabela 3: Parâmetros dos espectros Raman obtidos após aplicar as curvas de ajustes.

\begin{tabular}{|c|c|c|c|c|c|c|c|c|c|}
\hline \multirow[b]{2}{*}{ AMOSTRA } & \multicolumn{2}{|c|}{ RAMAN SHIFT } & \multicolumn{2}{|c|}{ LARGURA DE BANDA } & \multicolumn{2}{|c|}{ INTENSIDADE } & \multicolumn{2}{|c|}{ ÁREA } & \multirow[b]{2}{*}{$I_{D} / I_{G}$} \\
\hline & $\mathrm{D}$ & $\mathrm{G}$ & $\mathrm{FWHM}_{\mathrm{D}}$ & $\mathrm{FWHM}_{\mathrm{G}}$ & $\mathrm{D}$ & $\mathrm{G}$ & $\left(\mathrm{I}_{\mathrm{D}}\right)$ & $\left(\mathrm{I}_{\mathrm{G}}\right)$ & \\
\hline TiCNI & 1345,3 & 1584,2 & 269,4 & 101,8 & 1060 & 673,1 & 262442,05 & 37750,06 & 6,95 \\
\hline TiCNII & 1328,9 & 1580,9 & 271,4 & 93,7 & 1238,1 & 842,7 & 361517,45 & 67745,62 & 5,34 \\
\hline TiCNIII & 1330 & 1581,1 & 233,7 & 91,9 & 1748,2 & 1209,8 & 473546,2 & 110770,96 & 4,27 \\
\hline
\end{tabular}




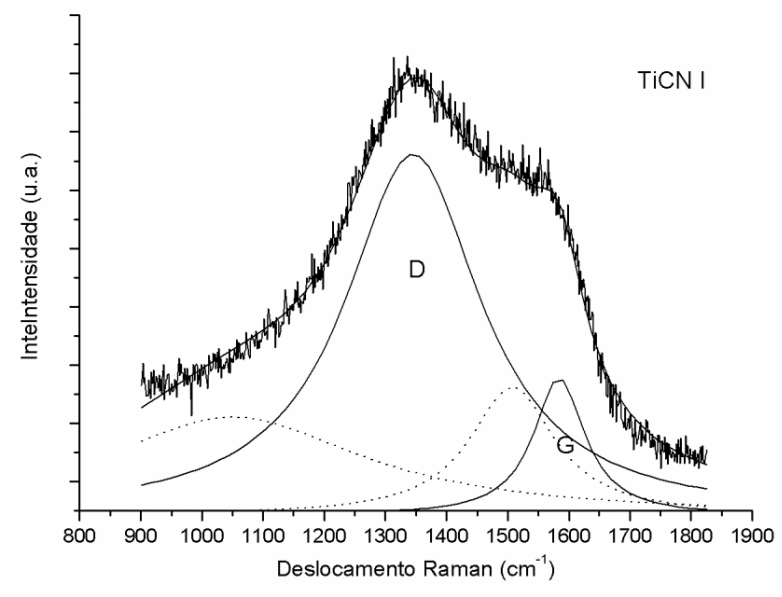

Figura 5: Espectro micro-Raman com as correspondentes curvas (Lorentzianas) de ajustes.

O perfil topográfico foi avaliado usando uma imagem numa área $5 \mu \mathrm{m} x \mu \mathrm{m}$. A amostra padrão apresenta uma superfície lisa, com uma rugosidade inicial de apenas $0,5 \mathrm{~nm}$ de perfil uniforme. Após o tratamento termoquímico de carbonitretação por plasma o perfil topográfico foi alterado. Os parâmetros de rugosidade (Ra, $\mathrm{Rz}$, Rp e Rv) foram crescentes com o aumento da proporção de $\mathrm{CH}_{4}$ no plasma carbonitretante (figura 6). Notar que as imagens não estão na mesma escala. A tabela 4 exibe esses parâmetros. Essa evolução poderia ser relacionada à presença de aglomerados de carbono, como tem sido relatado na literatura [25] para clusters de carbono com aspecto colunar distribuído de forma aleatória na superfície. Por outro lado também a formação de nitretos em $500^{\circ} \mathrm{C}$ promove um aumento da rugosidade [26].

Tabela 4: Tabela de parâmetros de rugosidade das amostras carbonitretadas e oxinitretadas em plasma.

\begin{tabular}{cccccc}
\hline AMOSTRA & $\mathbf{R a}(\mathbf{n m})$ & $\mathbf{R z}(\mathbf{n m})$ & $\mathbf{R p}(\mathbf{n m})$ & $\mathbf{R v}(\mathbf{n m})$ & $\mathbf{R p} / \mathbf{R z}$ \\
\hline TiCN I & 8,5 & 106,9 & 71,9 & 35,0 & 0,7 \\
TiCN II & 14,5 & 154,0 & 80,7 & 73,3 & 0,5 \\
TiCN III & 28,2 & 267,0 & 147,2 & 119,8 & 0,6 \\
Ti cp & 0,5 & 10,7 & 8,8 & 1,9 & 0,8 \\
\hline
\end{tabular}

A relação $R p / R z$ é de interesse por fornecer informações da topografia da superfície. Quando a razão Rp:Rz $>0,5$, a superfície tem pontas agudas e quando $\mathrm{Rp} / \mathrm{Rz}<0,5$ os picos são arredondados, conforme WHITEHEAD (1998) [27]. A amostra padrão apresentou $\mathrm{Rp} / \mathrm{Rz}=0,81$ enquanto que os substratos tratados em plasma apresentaram o valor entre 5,5 - 6,7. Isso sugere uma topografia com perfil ponti-agudo. CHENG e ZHENG [5] usaram $\mathrm{CH}_{4}, \mathrm{~N}_{2}$ e Ar para fabricar TiCN, aplicando uma relação $\mathrm{CH}_{4} / \mathrm{Ar}$ menor que 0.2 e obtiveram um filme sem excesso de carbono na superfície e com rugosidade Ra igual a 1,5 nm.

A dureza superficial das amostras TiCNI e TiCNII é maior próximo da superfície e depois decresce até um patamar por volta de 1,8 GPa, referente ao substrato (figuras 7). A dureza máxima destas amostras foi de 3,4 GPa e 2,4 GPa respectivamente, atribuídas à presença de TiN, de acordo com a análise de GIXRD. A amostra TiCN III exibe dureza inferior à do substrato. A literatura revela que a dureza superficial de TiCN aumenta linearmente com a concentrações de carbono em relação à soma entre carbono e nitrogênio: $[\mathrm{C}] /([\mathrm{C}]+[\mathrm{N}])$ [28]. Como neste trabalho se observou a formação de carbono na superfície, a formação de carbetos foi reduzida, alterando os valores de dureza do revestimento. A dureza diminuiu quando ocorreu a formação desse filme. Podemos ver que as amostras que apresentaram maior dureza foram aquelas que exibiram maior presença de TiN e menor pico de carbono grafite nos espectros Raman, obtidas com o menor fluxo de $\mathrm{CH}_{4}$. 


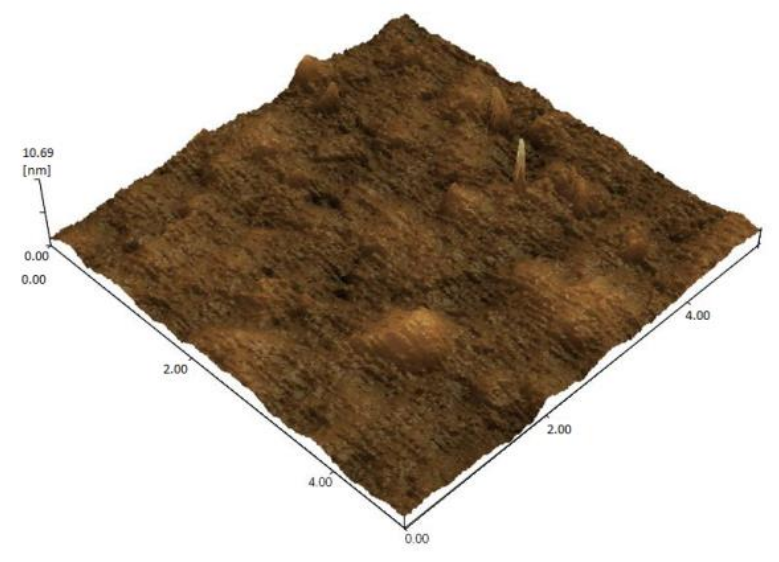

(a)

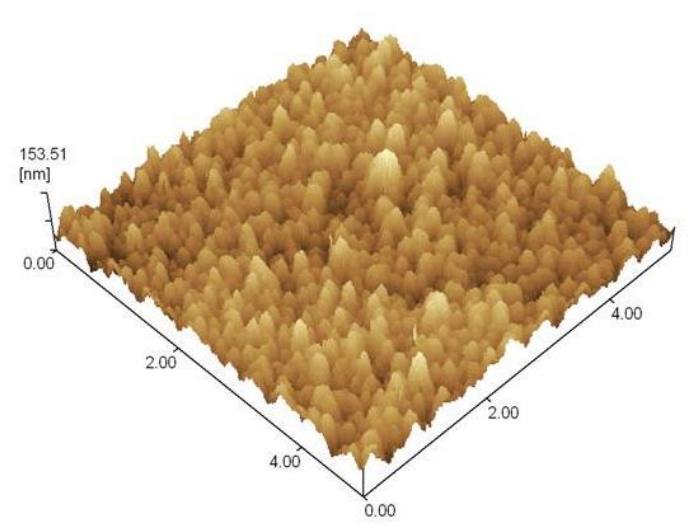

(c)

Figura 6: Imagens de AFM numa área $5 \mu \mathrm{m}$ x $5 \mu \mathrm{m}$ das amostras: titânio padrão (a), TiCN I (b), TiCN II (c) e TiCN III (d). A escala em "z" é distinta e está indicada nas quatro imagens, sendo máxima pra TiCN III.

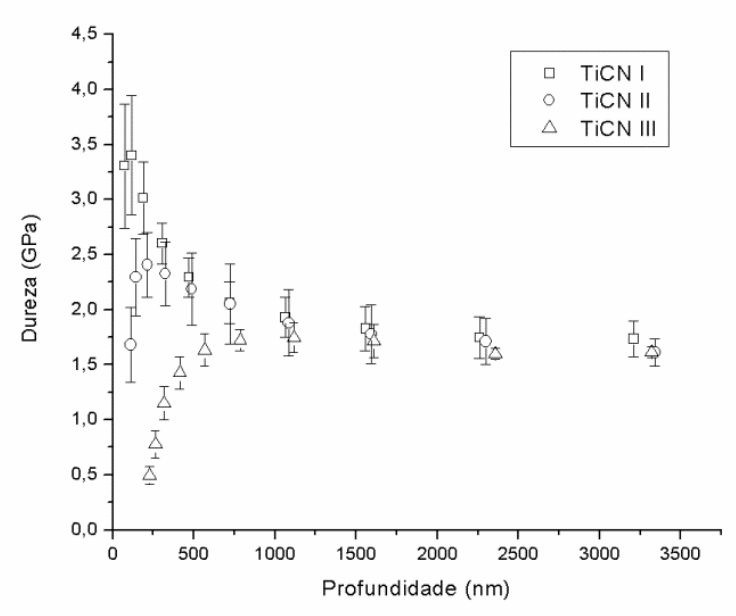

Figura 7: Dureza superficial de TiCN I-II em função da profundidade.

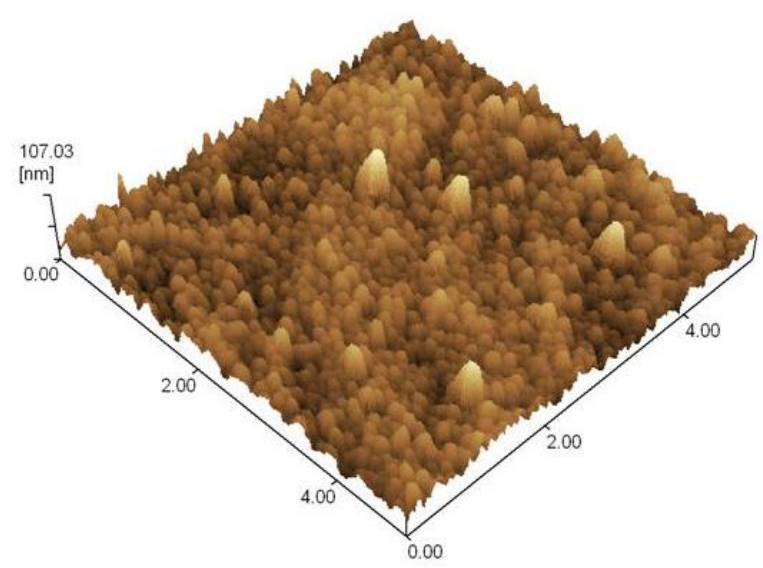

(b)

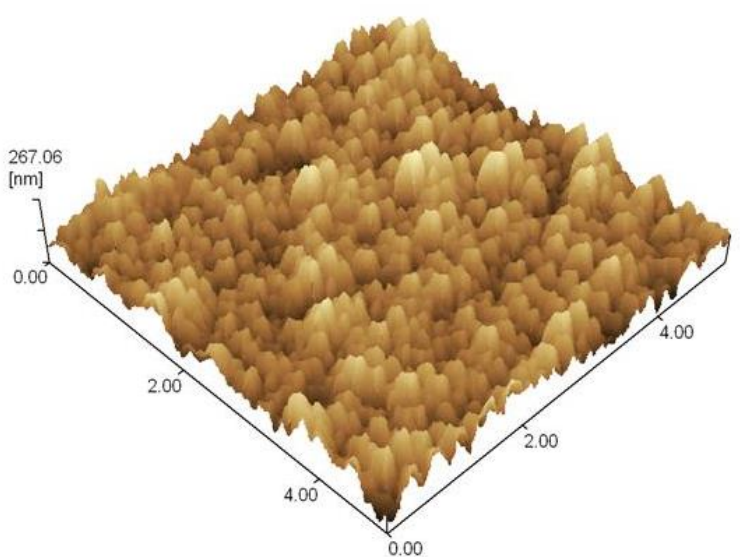

(d) 


\section{DISCUSSÕES}

Observando os dados da análise de GIXRD, nota-se que o teor da fase TiCN aumentou com o aumento da intensidade de emissão de $\mathrm{CN}$ no plasma, enquanto o pico da fase TiN decresceu junto com o sinal de $\mathrm{N}_{2}{ }^{+}$. Como a intensidade de emissão dessas espécies está relacionada com a concentração das mesmas, constatouse que seu teor influi na composição química superficial dos substratos. Observou-se também alargamento dos picos nos padrões de GIXRD referentes aos planos (111) e (222) do TiN e TiC.

Com a espectroscopia micro-Raman, foi possível detectar a formação de um filme fino de carbono na superfície de algumas amostras. VANDEVELDE et al. [17] mencionaram que a adição de nitrogênio aumenta a concentração de radicais de carbono no plasma e que esses radicais provavelmente são os responsáveis pela formação do carbono amorfo na superfície. A redução da relação entre as intensidades integradas das bandas $\mathrm{D}$ e $\mathrm{G}\left(\mathrm{I}_{\mathrm{D}} / \mathrm{I}_{\mathrm{G}}\right)$ é também indicativa da presença de carbono de baixa cristalinidade [르. A amostra TiCN I apresentou o carbono mais desordenado, com mais defeitos estruturais. O TiCN II apresentou menor deslocamento das bandas $\mathrm{D}$ e G que TiCN I $\left(1328,87 \mathrm{~cm}^{-1}\right.$ e $1580,87 \mathrm{~cm}^{-1}$ respectivamente). A largura à meia altura da banda $\mathrm{G}$ tende a diminuir, enquanto que a intensidade dessa banda aumenta quando a emissão de $\mathrm{CN}$ aumenta. De maneira geral, foi observado que a $\mathrm{I}_{\mathrm{D}}, \mathrm{I}_{\mathrm{G}}$ e as intensidades integradas evoluíram com o aumento da intensidade de $\mathrm{CN}$ enquanto que a largura da meia altura e $\mathrm{I}_{\mathrm{D}} / \mathrm{I}_{\mathrm{G}}$ diminuem. Isso sugere um crescimento do filme de carbono e um rearranjo estrutural do mesmo quando o sinal de $\mathrm{CN}$ evolui.

Quanto às propriedades superficiais, a topografia das amostras apresentam valores crescentes de rugosidade e um perfil topográfico pontiagudo que a amostra padrão, enquanto que a dureza diminuiu com o aumento da intensidade da espécie $\mathrm{CN}$ e a redução de $\mathrm{N}_{2}{ }^{+}$. O crescimento do filme de carbono parece ter sido a causa desse fenômeno, contaminado a superfície e evitando que o $\mathrm{N}$ difundisse para o interior da matriz de $\mathrm{Ti}$ (menor pico de TiN no GIXRD), resultando em uma camada com baixa dureza na superfície, que sobe um pouco acima dos valores de dureza do núcleo, para depois cair para este valor. No caso da amostra TiCN III, a dureza perto da superfície $(\sim 1 \mu \mathrm{m})$ foi menor que a do substrato

\section{CONCLUSÕES}

Este trabalho apresenta um estudo da correlação dos padrões da emissão das espécies $\mathrm{N}_{2}{ }^{+}, \mathrm{H}, \mathrm{C}, \mathrm{NH}, \mathrm{N}_{2} \mathrm{e}$ $\mathrm{CN}$ em relação à composição de fases e às propriedades superficiais (topografia e o perfil de microdureza) de camadas de TiCN. Foi observado que o plasma quebra as ligações do $\mathrm{CH}_{4}$ e se formam produtos como $\mathrm{NH}$, $\mathrm{H}, \mathrm{C}$ e CN . As espécies $\mathrm{CN}$ e $\mathrm{N}_{2}{ }^{+}$foram as que apresentam melhor correlação com a formação da camada de TiCN na superfície. A análise de GIXRD revelou que a inserção de $\mathrm{C}$ e $\mathrm{N}$ na superfície foi correlacionada com as variações de intensidade das espécies estudadas, revelando fases de nitreto e carbonitreto de titânio com composição química variável. Foi encontrado uma relação entre a queda da intensidade de emissão da espécie $\mathrm{N}_{2}{ }^{+} \mathrm{e}$ a redução do pico da fase TiN no difratograma e entre o aumento do sinal do $\mathrm{CN}$ com o aumento do pico de TiCN. As características topográficas foram influenciadas pela presença de um filme de carbono, com rugosidade crescente correlacionado com o sinal de carbono na espectroscopia micro-Raman e com aumento do sinal de emissão óptica de $\mathrm{CN}$ no plasma. O aumento da emissão de $\mathrm{CN}$ é provavelmente o responsável pela redução do teor da fase de TiN (GIXRD) e pelo aumento da camada de carbono, refletindo na redução progressiva da dureza superficial e aumento nos parâmetros de rugosidade.

\section{AGRADECIMENTOS}

Os autores agradecem à Coordenação de Aperfeiçoamento de Pessoal de Nível Superior (CAPES), à Fapergs e ao CNPq.

\section{BIBLIOGRAFIA}

[1] GHARESHABANI, E., et al., "Synthesis of nanostructure multiphase Ti(C,N)/a-C films by plasma focus device", Nuclear Instruments and Methods in Physics Research B, v. 268, pp. 2777-2784, Jun. 2010.

[2] DONG, Y.X., et al., "Characterization and blood compatibility of TiCxN1-x hard coating prepared by plasma electrolytic carbonitriding”, Surface \& Coatings Technology, v. 201, pp 8789-8795, Jun. 2007.

[3] SERRO, A.P., et al., "A comparative study of titanium nitrides, TiN, TiNbN and TiCN, as coatings for biomedical applications", Surface \& Coatings Technology, v. 203, pp 3701-3707, Jun. 2009.

[4] SU, Y.L., et al., "Comparison of tribological behavior of three films - TiN, TiCN and $\mathrm{CrN}$ - grown by physical vapor deposition”, Wear, v. 213, pp. 165-174, Jun 1997. 
[5] CHENG, Y., ZHENG, Y.F., "Characterization of TiN, TiC and TiCN coatings on Ti-50.6 at \% Ni alloy deposited by PIII and deposition technique", Surface \& Coatings Technology, v. 201, pp. 4909-4912, Ago. 2006.

[6] YANG, Y. et al., "Microstructure and wear properties of TiCN/Ti coatings on titanium alloy by laser cladding", Optical and Lasers in Engineering, v. 48, pp. 119-124, Set. 2009.

[7] KARLSSON, L., et al., "Growth, microstructure, and mechanical properties of arc evapored $\operatorname{TiC}_{\mathrm{x}} \mathrm{N}_{1-\mathrm{x}}$ $(0<\mathrm{x}<1)$ films", Surface \& Coatings Technology, v. 126, pp. 1-14, Jan 2000.

[8] RIE, K.-T., WHOLE, J., GEBAUER, A. "Low temperature coating of aluminum alloys by plasma-CVD using metallo-organic compounds", Surface \& Coatings Technology, v. 98, pp. 1534-1540, 1998.

[9] RIE, K.-T. "Recent advances in plasma diffusion processes", Surface \& Coatings Technology, v.112, pp. 56-62, 1999.

[10] RIE, K.-T., WHOLE, J., "Plasma-CVD of TiCN and ZrCN films on light metals", Surface \& Coatings Technology, v. 112, pp. 226-229, 1999.

[11] KULAKOWSKA-PAWLAK, B. ZYRNICKI, W., "Characterization of a d.c. titanium tetrisopropoxide/H2/N2 plasma using emission spectroscopy", Thin Solid Films, v. 266, pp. 8-13, Abril 1995.

[12] BARAVIAN, G., et al., "Optical emission spectroscopy of active species in a TiCN PVD arc discharge", Surface \& Coatings Technology, v. 76-77, 687-693, 1995.

[13] BARHOLM-HANSEN, C, et al., "Process control by optical emission spectroscopy during growth of a$\mathrm{C}: \mathrm{H}$ from a $\mathrm{CH}_{4}$ plasma by plasma-enhanced chemical vapour deposition", Surface \& Coatings Technology, v. 68-69, pp. 702-707, 1994.

[14] National Institute of Standards and Technology (NIST), http://www.nist.gov/pml/data/asd.cfm. Acessado em dezembro de 2012.

[15] PEARSE, R.W.B., GAYDON, A.G., The indentification of molecular spectra, 4 ed., London, Chapman and Hall, 1976.

[16] JAMROZ, P., ZYRNICKI, W., "Optical emission spectroscopy study for nitrogen-acetylene-argon and nitrogen-acetylene-helium $100 \mathrm{kHz}$ and dc discharges", Vacuum, v. 84, pp. 940-946, Dez. 1999.

[17] VANDEVELDE, T., et al., "Correlation between the OES plasma composition and the diamond film properties during microwave PA-CVD with nitrogen addition", Thin Solid Films, v. 340, pp. 159-163, Set. 1998.

[18] LEGRAND, J-C., et al., "Kinetics of reactions in $\mathrm{CH}_{4} / \mathrm{N}_{2}$ afterglow plasma a simplified model", Vacuum, v. 50, n. 3-4, pp. 491-495, Jan. 1998.

[19] SCHNEIDER, J.M., et al., "X-ray diffraction investigations of magnetron sputtered TiCN coatings", Surface \& Coatings Technology, v. 74-75, pp. 312-319, 1995.

[20] SOLTANI-FARSHI, M., et al., "Content of hydrogen in boron-, carbono, nitrogen-, oxygen-, fluorineand neon-implanted titanium", Surface \& Coatings Technology, v. 103-104, pp. 299-303, 1998.

[21] LOBO, A.O., et al., "Caracterização de materiais carbonosos por espectroscopia Raman”, Revista Brasileira de Aplicações de Vácuo, v. 24, n. 2, pp. 98-103, Out. 2005.

[22] PIMENTA, M.A., et al., "Studying disorder in graphite-based systems by Raman spectroscopy", Physical Chemistry Chemical Physics, v. 9, pp. 1276-1291, Jan. 2007.

[23] LOHSE, B.H., CALKA, A., WEXLER, D., "Raman spectroscopy sheds new light on TiC formation during the controlled milling of titanium and carbon", Journal of Alloys and Compounds, v. 434-435, pp. 405-409, Out. 2006.

[24] ZAIDA, A., et al., "Further development of Raman Microprobe spectroscopy for characterization of char reactivity", Proceeding of the Combustion Institute, v. 31, pp. 1881-1887, 2007.

[25] OSSI, P.M., MIOTELLO, A., "Control of cluster synthesis in nano-glassy carbon films", Journal of Non-Crystalline Solids, v. 353, pp. 186-1864, Mar. 2007.

[26] VASCONCELLOS, M.A.Z., et al., "The correlation temperature and atmosphere on the plasma properties and on the surface hardness and roughness of D.C. plasma nitrided Ti-6Al-4V alloy", submetido para Thin Surface and Coatings, 2014.

[27] WHITEHEAD, S.A., et al., "Comparison of two stylus methods for measuring surface texture", Dental Materials, v. 15, pp. 79-86, Dez. 1998. 
NUNES FILHO, A.;BRAZ, D. C.; HINRICHS, R.; VASCONCELLOS, M.A.Z.; ROCHA, R.C.S.; ALVES JUNIOR, C. revista Matéria, v.20, n.1, pp. 72-82, 2015.

[28] YANG, Q., et al., "Hardness and elastic properties of $\operatorname{Ti}\left(\mathrm{C}_{\mathrm{x}} \mathrm{N} 1_{-\mathrm{x}}\right), \operatorname{Zr}\left(\mathrm{C}_{\mathrm{x}} \mathrm{N}_{1-\mathrm{x}}\right)$ and $\mathrm{Hf}\left(\mathrm{C}_{\mathrm{x}} \mathrm{N}_{1}-\mathrm{x}\right)$ ", Journal of Alloys and Compounds, v. 309, pp. L5-L9, Mai. 2000. 